\title{
Electroacupuncture protects against ischemic stroke by reducing autophagosome formation and inhibiting autophagy through the mTORC1-ULK1 complex-Beclin1 pathway
}

\author{
WEILIN LIU ${ }^{1}$, GUANHAO SHANG ${ }^{2}$, SHANLI YANG $^{3}$, JIA HUANG $^{1}$, XIEHUA XUE $^{3}$, YUNJIAO LIN $^{2}$, \\ YI ZHENG ${ }^{2}$, XIAN WANG ${ }^{2}$, LULU WANG ${ }^{2}$, RUHUI LIN ${ }^{2}$, JING TAO $^{1}$ and LIDIAN CHEN ${ }^{1}$ \\ ${ }^{1}$ College of Rehabilitation Medicine, Fujian University of Traditional Chinese Medicine, Fuzhou, Fujian 350122; \\ ${ }^{2}$ The Fujian Province Key Laboratory of Motor Functional Rehabilitation; ${ }^{3}$ Rehabilitation Hospital Affiliated to \\ Fujian University of Traditional Chinese Medicine, Fuzhou, Fujian 350001, P.R. China
}

Received July 16, 2015; Accepted November 12, 2015

DOI: $10.3892 / \mathrm{ijmm} .2015 .2425$

\begin{abstract}
In a previous study by our group, we demonstrated that electroacupuncture (EA) activates the class I phosphoinositide 3-kinase (PI3K)/Akt signaling pathway. There is considerable evidence that the downstream mammalian target of rapamycin complex 1 (mTORC1) plays an important role in autophagy following ischemic stroke. The aim of the present study was to determine whether EA exerts a neuroprotective effect through mTORC1-mediated autophagy following ischemia/reperfusion injury. Our results revealed that EA at the LI11 and ST36 acupoints attenuated motor dysfunction, improved neurological deficit outcomes and decreased the infarct volumes. The number of autophagosomes, autolysosomes and lysosomes was decreased following treatment with EA. Simultaneously, the levels of the autophagosome membrane maker, microtubule-associated protein 1 light chain 3 beta (LC3B)II/I, Unc-51-like kinase 1 (ULK1), autophagy related gene 13 Atg13) and Beclin1 (ser14) were decreased, whereas mTORC1 expression was increased in the peri-infarct cortex. These results suggest that EA protects against ischemic stroke through the inhibition of autophagosome formation and autophagy, which is mediated through the mTORC1-ULK complex-Beclin1 pathway.
\end{abstract}

\section{Introduction}

Stroke, also known as cerebrovascular disease, is the second leading cause of mortality, accounting for hundreds of thousands of mortalities annually worldwide, and is a major cause

Correspondence to: Professor Lidian Chen or Professor Jing Tao, College of Rehabilitation Medicine, Fujian University of Traditional Chinese Medicine, 1 Qiuyang Road, Fuzhou, Fujian 350122, P.R. China E-mail: cld@fjtcm.edu.cn

E-mail: 369049101@qq.com

Key words: electroacupuncture, autophagy, mammalian target of rapamycin complex 1, Unc-51-like kinase complex, Beclin1 of disability in adults (1). Ischemic stroke due to the lack of blood flow is more prevalent than hemorrhagic stroke due to bleeding. Within the ischemic core of the brain, where blood flow is most severely restricted, excitotoxic and necrotic cell death occurs within minutes. In cases of prolonged ischemia, hypoxanthine is formed as a product of adenosine triphosphate (ATP) metabolism (2). However, rapid revascularization of the occluded vessels and the restoration of blood flow in cases of acute ischemic stroke may cause ischemia/reperfusion (I/R) injury (3). An imbalance in the energy supply and demand within the ischemic brain tissue results in surrounding tissue hypoxia and dysfunction. Subsequent reperfusion further enhances the activation of innate and adaptive immune responses and cell death programs (4).

The classic forms of cell death include necrosis, apoptosis and autophagy (6) Autophagy, or cellular self-digestion, is an evolutionarily highly conserved catabolic process that is important for balancing sources of energy at critical time points in development and in response to nutrient stress, and has been proposed as the third type of cell death (5-7). Autophagy is constitutively active in the central nervous system (CNS), and protects against neuronal injury and neurodegeneration following ischemic stroke $(8,9)$. In early cerebral ischemia, autophagy can degrade damaged organelles, eliminate I/R-induced damaged components and toxic metabolites or provide the nutrient source required to maintain metabolism, ATP levels, cellular homeostasis and survival (10-12).

Macroautophagy is the main pathway involved in autophagy, and it is used mainly to eradicate damaged cell organelles or unused proteins (13). In canonical macroautophagy, a small part of the cytoplasm is sequestered by a membrane sac, the so-called isolation membrane (also termed the phagophore), which results in the formation of a double-membrane structure, the autophagosome. The autophagosome matures as it fuses with endosomes and then finally fuses with lysosomes. Following fusion, the inner membrane and enclosed cytoplasmic materials are degraded by lysosomal enzymes (14). In the canonical ischemia-induced pathway, autophagosome formation is regulated by autophagy-related gene (Atg) 8 [also known as light chain 3 (LC3)-phosphatidylethanolamine conju- 
gate (LC3-II)], and the mammalian target of rapamycin (mTOR) complex 1 (mTORC1) signaling pathway (15-18). Under nutrient-rich conditions, the upregulation of mTORC1 leads to the inactivation of the Atg1/Unc-51-like kinase (ULK) complex that consists of ULK1, ULK2, Atg13 and FIP200 in mammals (19). Under starvation conditions, the ULK complex is involved in the initial step of autophagosome formation, which is activated by inactivated mTORC1 $(20,21)$. The ULK complex promotes autophagy by targeting the autophagosome formation-specific class III phosphoinositide 3-kinase (PI3K) complex which consists of class III PI3K, Atg14L (also known as Atg14 and Barkor), Beclin1 (Atg6 homolog), hVps15 and hVps34 $(22,23)$.

However, it has been previously noted that the excessive activation of autophagy may promote cell autophagic death (24). The overactivation of autophagy causes neuronal cells to self-digest their own necessary components or interact with the apoptotic cascade, thus promoting nerve cell death in the ischemic surrounding zones in cerebral ischemia (25-27). We, as well as others have demonstrated that electroacupuncture (EA) at the LI11 or ST36 acupoints activats the class I PI3K/Akt signaling pathway (28-30). However, activated Akt leads to the activation of downstream mTORC1 in the modulation of autophagy $(31,32)$. Therefore, we concluded that EA at the LI11 and ST36 acupoints induced the expression of mTORC1 in ischemic stroke. Thereby, a study hypothesis was proposed that EA protects against ischemic stroke through the mTORC1-ULK1 complex-Beclin1 pathway-mediated regulation of autophagosome formation and autophagy in the peri-infarct cortex following cerebral middle cerebral artery occlusion and reperfusion $(\mathrm{MCAO} / \mathrm{R})$ injury.

\section{Materials and methods}

Experimental animals and groups. Healthy male SpragueDawley (SD) rats, weighting $280 \pm 20 \mathrm{~g}$, were provided by Shanghai Laboratory Animal Co., Ltd., (SLAC) Shanghai, China (license no. SCXK 2014-0005) and housed under pathogen-free conditions with a 12 -h light/dark cycle, at $23 \pm 2^{\circ} \mathrm{C}$ and $60-70 \%$ humidity. The rats were allowed free access to food and water. All experimental protocols were approved by the Committee on Animal Care and Usage of Fujian University of Traditional Chinese Medicine (FUTCM), and all the principles in the Chinese Specifications for the Care and Use of the Laboratory Animals (SPF animal laboratory) were taken into account. The animals were subjected to adaptive feeding at the Laboratory Animal Center of FUTCM [license no. SYXK(min) 2014-007]. All efforts were made to minimize animal suffering.

According to the random number table method, $45 \mathrm{SD}$ rats were randomly and evenly divided into 3 groups $(n=15)$ as follows: i) the sham-operated control group (sham group); ii) the $\mathrm{MCAO} / \mathrm{R}$ control group; and iii) the $\mathrm{MCAO} / \mathrm{R}+\mathrm{EA}$ treatment group (MCAO/R + EA group).

Establishment of the rat model of MCAO/R injury. The rat model of MCAO/R injury was established using the sutureoccluded method. The detailed procedure has previously been described in the study by Longa et al (33). Briefly, the rats were anesthetized with $10 \%$ chloral hydrate $(300 \mathrm{mg} / \mathrm{kg}$ body weight) by intraperitoneal injection. MCAO on the left side was performed using an occluding suture with an embolus (Jia Ling embolus; Jia Ling Biological Technology Ltd., Guangzhou, China) for $2 \mathrm{~h}$, and after $2 \mathrm{~h}$ of MCAO-induced cerebral ischemia, the suture was slowly withdrawn to allow for reperfusion. The ipsilateral cerebral blood flow (CBF) was measured by Laser Doppler Flowmetry (Biopac Systems, Goleta, CA, USA). The MCAO model was considered successful (inclusion) only when CBF dropped to become equal or $>80 \%$ of the baseline during occlusion. The rectal temperature of the rats was maintained at $37^{\circ} \mathrm{C}$ throughout the surgical procedures. In the sham group, the artery was isolated, but no embolus was introduced. After surgery and recovery, the animals were placed in an environment at room temperature, and they resumed a normal diet.

Assessment of neurological deficit scores. Neurological function was assessed in each rat $2 \mathrm{~h}$ after MCAO/R injury and 3 days after EA treatment in a blinded manner using a previously described method (33). Detailed scores are as follows: score 0 , no neurological deficit; score 1 , the tail was raised and adduction (not able to fully extend) of the right forelimb was observed; score 2, spontaneous circling to the right when walking; score 3 , the body was slanted to the right when walking; score 4 , not able to walk spontaneously along with possible loss of consciousness. Scores of 0 and 4 indicated modeling failure, and rats with these scores were excluded.

Treatment with EA. The rats in the MCAO/R + EA group received EA treatment. Stainless acupuncture needles $(0.3 \mathrm{~mm}$ in diameter, Huatuo acupuncture needle; Suzhou Medical Appliance Factory, Suzhou, China) were inserted 2-3 mm deep into the Quchi (LI11, located in the depression lateral to the anterior aspect of the radius joint of the forelimb) and Zusanli (ST36, located $5 \mathrm{~mm}$ below the knee joint of the hind-limb and $2 \mathrm{~mm}$ lateral to the anterior tubercle of the tibia) acupoints on the right paralyzed limb. Stimulation was then generated by an electroacupuncture instrument [model G6805; Shanghai Marine Instrument General Factory (SMIF), Shanghai, China], and the stimulation parameters were set as dense disperse waves of $1-20 \mathrm{~Hz}$ (adjusted to the muscle twitch threshold); peak voltage of $6 \mathrm{~V}, 0.2 \mathrm{~mA}$ intensity for $30 \mathrm{~min} / \mathrm{day}$, once a day. EA treatment was administered $24 \mathrm{~h}$ after MCAO/R and continued until the animals were sacrificed with $10 \%$ chloral hydrate (Fujian Academy of Integrative Medicine, FUTCM) anesthesia after EA treatment, which ran consecutively for 3 days.

Measurement of the infarct volume. At the end of the neurological examination, the brains were quickly removed to measure the infarct volume, as previously described (34). The rat brains were rapidly dissected after the were anesthetized using $10 \%$ chloral hydrate (Fujian Academy of Integrative Medicine, FUTCM), fresh brain tissue was placed in a freezer at $-80^{\circ} \mathrm{C}$ for $20 \mathrm{~min}$ and 2-mm coronal sections were cut. The fresh slices were immediately immersed in 2\% 2,3,5-triphenyltetrazolium chloride (Sigma-Aldrich, St. Louis, MO, USA). The TTC-stained sections were photographed using a high-resolution digital camera (Canon SX20), and the infarct area was determined with computerized image analysis software (Motic Med 6.0 system). The total infarct volume was analyzed using 6 slices of 2-mm coronal sections from each brain and calculated using the 
following formula: infarct volume $(\%)=$ (infarct volume/total volume) $\mathrm{x} 100$. The percentage brain infarct volume was used for subsequent statistical analysis.

Computer-assisted method for gait analysis. We used CatWalk (Noldus Information Technology, Wageningen, The Netherlands), a quantitative and automated gait analysis system, which measures numerous parameters of gait. Previous studies using CatWalk have identified multiple $\mathrm{MCAO} / \mathrm{R}$-sensitive gait parameters which can be utilized to assess the gait and motor function in murine models of $\mathrm{MCAO} / \mathrm{R}$ injury (35-37). The 'CatWalk' apparatus is made of a 1.3-m-long glass platform which is illuminated from the long edge with green fluorescent lights which are completely internally reflected. When the rat walks along the walkway with paws in contact with the glass surface, the light is reflected downward and the images of the paw prints are captured by a high-speed video camera under the walkway. Images and data were analyzed using CatWalk XT 10.0 software. The animals received 7 days of training 6 times each day to familiarize them with the walkway prior to the operation, as previously described (38). There were 2 basic criteria: i) rats had to run across the walkway without any interruption, walking backwards or rearing during the run, and ii) at least 3 correct crossings per rat were required (39). All rats were subjected to gait assessment following treatment with EA for 3 days using the CatWalk automated gait analysis system.

Transmission electron microscopic examination. Following treatment with EA for 3 days, the rats were anesthetized and then perfused transcardially with pre-cooled phosphatebuffered saline (PBS; pH 7.4) followed by PBS containing $4 \%$ paraformaldehyde and $0.25 \%$ glutaraldehyde. The following day, the rat brains were sectioned with a vibratome (Leica Microsystems Ltd., Milton Keynes, UK) into 500- $\mu$ m-thick slices. The parietal lobe cortex of the peri-infarct cortical tissue and the corresponding area of the sham-operated rats were selected for analysis, and selected areas were processed by post-fixation in $1 \%$ osmium tetroxide and $1.5 \%$ potassium ferrocyanide for $2 \mathrm{~h}$, and they were then washed 3 times with PBS, dehydrated in graded ethanol and then embedded in epoxy resin (E51-618). Polymerization was performed at $37^{\circ} \mathrm{C}$ for $24 \mathrm{~h}$. Blocks were sectioned using a PowerTome PC ultramicrotome (RMC, Inc., Tucson, AZ, USA) into ultra-thin sections $(80 \mathrm{~nm})$, which were post-stained with uranyl acetate and lead citrate, and visualized using a Hitachi H-7650 electron microscope (Hitachi, Tokyo, Japan) at $80 \mathrm{kV}$. For quantitative analysis of the number of mitochondria, autophagosomes, autolysosomes and lysosomes, 3 rats per group and 10 random fields of vision for each rat were counted by a blinded observer.

Western blot analysis. Total proteins were extracted from the peri-infarct cortical tissue supplied by the rats which had been subjected to $\mathrm{MCAO} / \mathrm{R}$ on the left side and the corresponding area of sham-operated rats. Western blot analysis was carried out with $10-12 \%$ sodium dodecyl sulfate-polyacrylamide gel electrophoresis (SDS-PAGE). Equal amounts of protein $(25 \mu \mathrm{g})$ were loaded onto the gels for western blot analysis. Following electrophoresis, proteins were transferred onto polyvinylidene fluoride (PVDF) membranes (pore size, $0.45 \mu \mathrm{m}$; Millipore, Billerica, MA, USA), which were blocked with 5\% skim milk
Table I. Neurological deficit scores.

\begin{tabular}{lccc}
\hline Group & $\mathrm{n}$ & $\begin{array}{c}2 \mathrm{~h} \text { after } \\
\mathrm{MCAO} / \mathrm{R}\end{array}$ & $\begin{array}{c}3 \text { days after } \\
\text { EA treatment }\end{array}$ \\
\hline Sham & 15 & 0 & 0 \\
MCAO/R & 15 & $2.17 \pm 0.17$ & $1.75 \pm 0.18$ \\
MCAO/R + EA & 15 & $2.08 \pm 0.19$ & $1.42 \pm 0.19^{\mathrm{a}}$ \\
\hline
\end{tabular}

Sham, sham-operated control group; $\mathrm{MCAO} / \mathrm{R}$, middle cerebral artery occlusion and reperfusion control group; $\mathrm{MCAO} / \mathrm{R}+\mathrm{EA}$, $\mathrm{MCAO} / \mathrm{R}+\mathrm{EA}$ treatment group. Data are expressed as the means $\pm \mathrm{SEM}$ from 15 individual rats in each group. ${ }^{\mathrm{a}} \mathrm{p}<0.05$, the $\mathrm{MCAO} / \mathrm{R}+\mathrm{EA}$ group vs. the $\mathrm{MCAO} / \mathrm{R}$ group. EA, electroacupuncture.

for $2 \mathrm{~h}$ at room temperature. The mmbranes were washed in TBS with $0.25 \%$ Tween-20 (TBST), then probed with primary antibodies against LC3B (1:1,000 dilution; No. 3868, Cell Signaling Technology, Danvers, MA, USA), Beclin1 (ser14) (1:2,000 dilution; ab183335), Atg13 (1:10,000 dilution; ab105392), ULK1 (1:10,000 dilution; ab128859) (all from Abcam, Cambridge, MA, USA), mTORC1 (1:1,000 dilution; No. 2587, Cell Signaling Technology) and $\beta$-actin (1:8,000 dilution; HC201-02, TransGen Biotech, Beijing, China) at $4^{\circ} \mathrm{C}$ overnight, followed by incubation with the appropriate HRP-conjugated secondary antibody (1:5,000 dilution; No. 58203 and No. 7077, Cell Signaling Technology) for $2 \mathrm{~h}$ at room temperature. The membranes were then washed again in TBST. The antibody-bound protein bands were detected with an enhanced chemiluminescence kit, and images were analyzed using a Bio-Image Analysis System (BioRad, Hercules, CA, USA). $\beta$-actin was used as a loading control. The ratio of grayscale values of the target protein to the control was used to measure and stored for subsequent analysis.

Statistical analysis. The data are expressed as the means \pm standard error of mean (SEM) and analyzed using SPSS 18.0 software. Comparisons between 2 groups were performed using the independent two-sample t-test or Mann-Whitney U test, and comparisons between multiple groups were performed with one-way ANOVA. A p-value $<0.05$ was considered to indicate a statistically significant difference.

\section{Results}

Effects of EA on neurological deficits and infarct volumes. The neuroprotective effects of EA at the LI11 and ST36 acupoints were evaluated by neurological behavioral assessment and measurement of the cerebral infarct volume. As shown in Table I and Fig. 1, rats in the MCAO/R + EA group and the MCAO/R group all exhibited manifestations of neurological deficits and cerebral infarction, whereas the rats in the sham group did not exhibit any signs of cerebral injury, which indicated that the rat model of cerebral MCAO/R injury was successfully established. The neurological behavioral scores between the rats in the $\mathrm{MCAO} / \mathrm{R}+\mathrm{EA}$ group and those in the $\mathrm{MCAO} / \mathrm{R}$ group at $2 \mathrm{~h}$ after $\mathrm{MCAO} / \mathrm{R}$ injury (prior to EA treatment) did not differ significantly (Table I, $\mathrm{p}>0.05$ ). However, following treatment with EA for 3 days, the neurological behavioral scores and cerebral infarct volumes were all reduced significantly (Table I and 
A

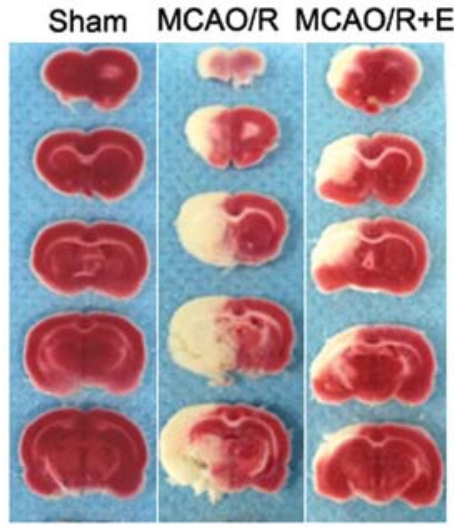

B

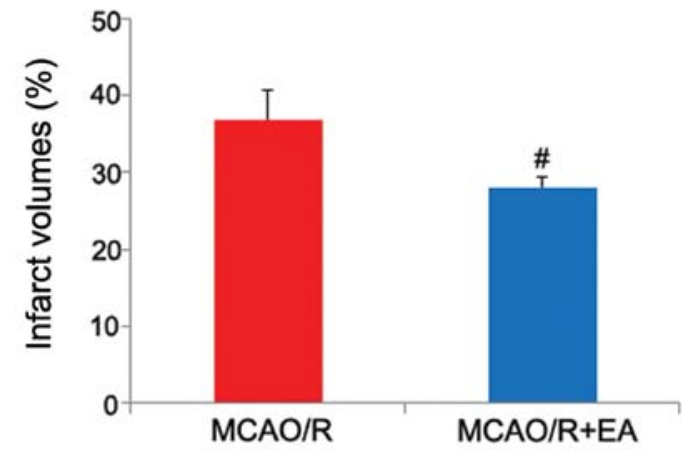

Figure 1. Electroacupuncture (EA) decreases the infarct volumes in rats with middle cerebral artery occlusion and reperfusion (MCAO/R) injury. (A) TTC staining indicating the cerebral infarct volume of Ssham, MCAO/R and MCAO/R + EA groups. (B) Bar graph shows the percentage total infarct volume in the rats with $\mathrm{MCAO} / \mathrm{R}$ ) injury. Data are expressed as the means \pm SEM from 4 individual rats in each group. ${ }^{\#}<0.05$, the $\mathrm{MCAO} / \mathrm{R}+\mathrm{EA}$ group vs. the $\mathrm{MCAO} / \mathrm{R}$ group.
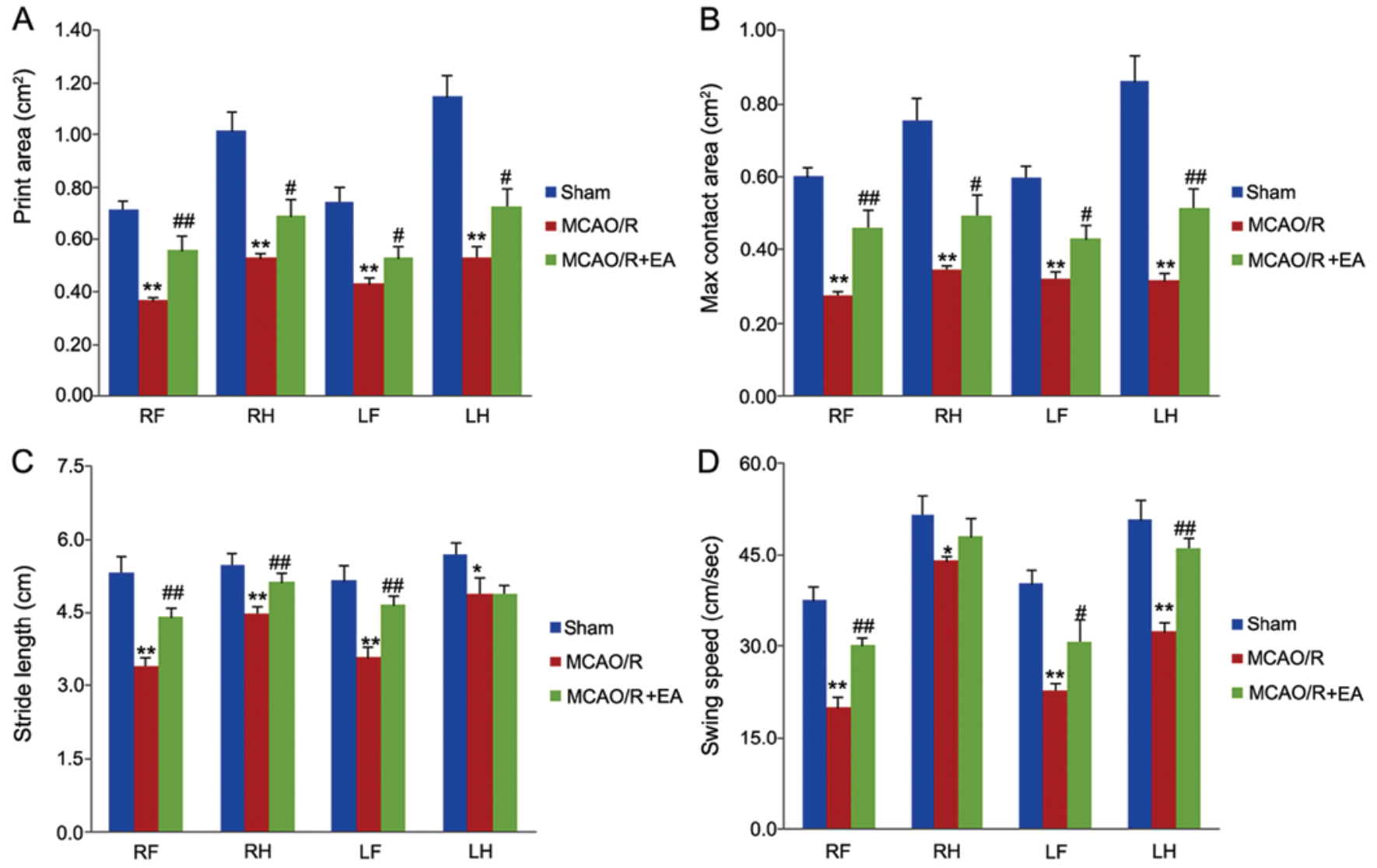

Figure 2. Electroacupuncture (EA) improves motor function in rats with middle cerebral artery occlusion and reperfusion (MCAO/R) injury. Motor function was assessed with a CatWalk gait analysis system using the (A) print area, (B) maximum contact area, (C) stride length and (D) swing speed, which was applied after EA treatment for 3 days. Data are expressed as the means \pm SEM from 15 individual rats in each group. ${ }^{* *} \mathrm{p}<0.01$ or ${ }^{*} \mathrm{p}<0.05$, the MCAO/R group vs. the sham group; ${ }^{\# \#} \mathrm{p}<0.01$ or ${ }^{\#} \mathrm{p}<0.05$, the MCAO/R + EA group vs. the MCAO/R group. RF, right fore; RH, right hind; LF, left fore; LH, left hind limbs.

Fig. 1; $\mathrm{p}<0.05)$. These results demonstrated that EA protected the rat brains against cerebral $\mathrm{MCAO} / \mathrm{R}$ injury.

Effects of EA on motor function. To examine the effects of EA treatment on motor function, the CatWalk automated gait analysis system was used following treatment with EA for 3 days. As shown in Fig. 2, following cerebral MCAO/R injury, in the rats in the $\mathrm{MCAO} / \mathrm{R}$ group, the print area, maximum contact area, stride length and swing speed of their 4 limbs were all decreased compared to those of the rats in the sham group $(\mathrm{p}<0.05$ or $\mathrm{p}<0.01)$. Following treatment with EA for 3 days, in the rats in the $\mathrm{MCAO} / \mathrm{R}+\mathrm{EA}$ group, the print area and maximum contact area of their 4 limbs, the stride length of their right fore (RF), right hind (RH) and left fore (LF) limbs, and the swing speed 

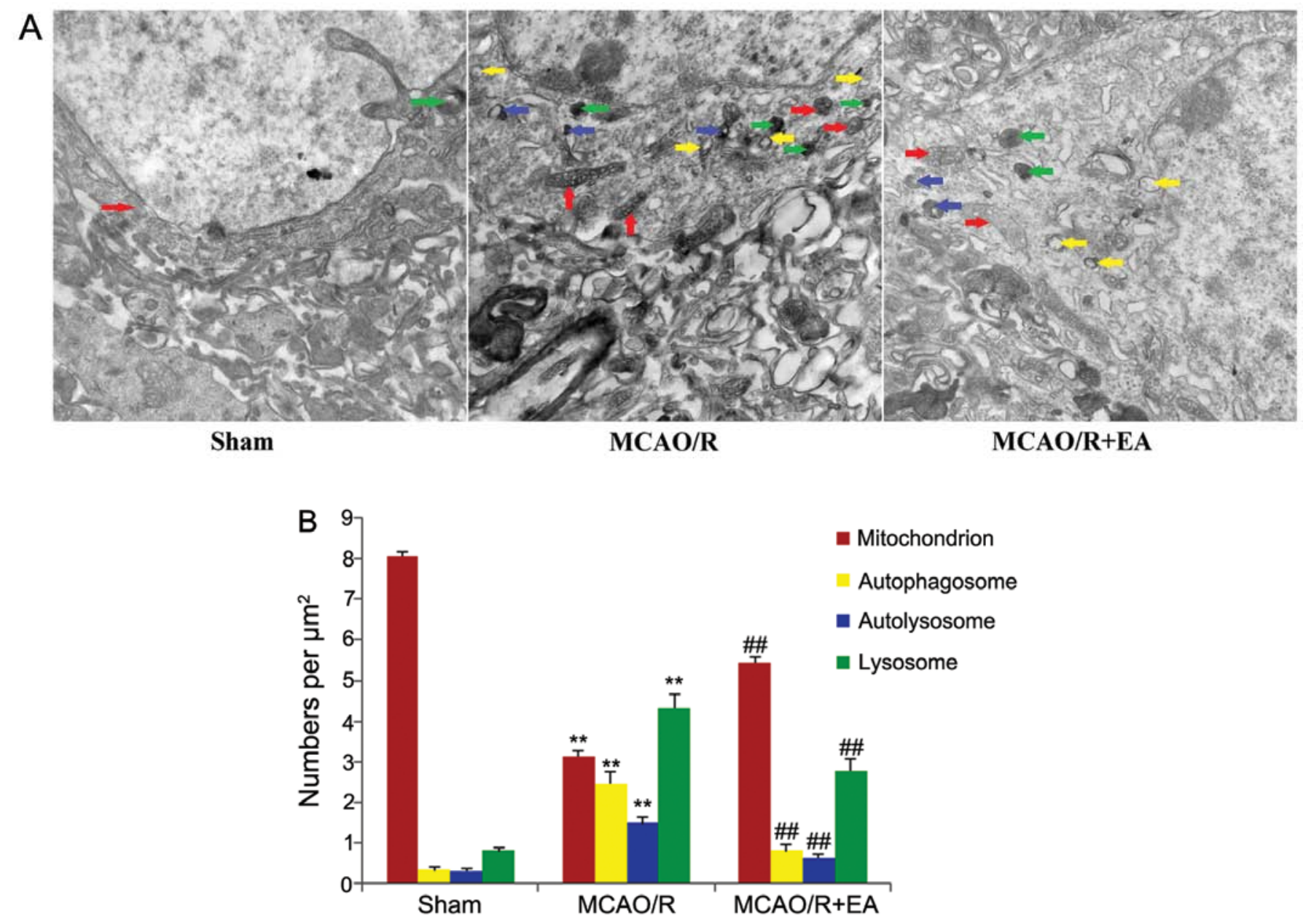

Figure 3. Effects of electroacupuncture (EA) treatment on ultrastructural changes in the peri-infarct cortex. (A) Sample transmission electron microscopy (TEM) images of cerebral cortex neurons. The sham-operated (sham) group, middle cerebral artery occlusion and reperfusion (MCAO/R) group, and MCAO/R + EA group are shown (x25,000 original magnification under TEM). Scale bars, $1 \mu \mathrm{m}$. Red arrows indicate mitochondria; yellow arrows indicate autophagosomes; blue arrows indicate autolysosomes; and green arrows indicate lysosomes. (B) Quantitative analysis of the number of normal mitochondria, autophagosomes, autolysosomes and lysosomes. Three rats in each group and 10 fields for each rat were examined. Data are expressed as the means \pm SEM. ${ }^{* *}$ p $<0.01$ vs. sham group; ${ }^{\# \#} \mathrm{p}<0.01$ vs. MCAO/R group.

of their RF, LF and left hind (LH) limbs were increased significantly, compared to the rats in the $\mathrm{MCAO} / \mathrm{R}$ group $(\mathrm{p}<0.05$ or $\mathrm{p}<0.01$. However, the stride length of their LH limbs, and the swing speed of their RH limbs in both groups were not altered significantly $(\mathrm{p}>0.05)$. These results suggest that EA at the LI11 and ST36 acupoints improves motor dysfunction in rats with cerebral MCAO/R injury.

Effects of EA on the morphological ultrastructural changes in the peri-infarct cortex. Transmission electron microscopy (TEM) is considered the gold standard for the detection of autophagic structures $(40,41)$. In order to further examine the effects of EA treatment on autophagy, we used TEM to observe the morphological ultrastructural changes of peri-infarct cortical neurons in each group. The neurons in the cerebral cortex of the rats in the sham group appeared to be relatively healthy, with normal polyribosomes, endoplasmic reticula, Golgi apparatus, mitochondria, lysosomes, nuclei and fine granular chromatin evenly distributed (Fig. 3A, sham group). By contrast, the cortical neurons in the peri-infarct area in the rats in the MCAO/R displayed morphological changes typical of infarction: the neurons exhibited a dilated endoplasmic reticulum, swollen and balloon-like mitochondria, mitochondrial crests which had fused or disappeared, and the formation of numerous vacuoles in the cytoplasm and numerous darkened lysosomes and autophagosomes (C-shaped double-membrane structures) were also observed (Fig. 3A, MCAO/R group). The number of normal mitochondria was decreased in the rats in the $\mathrm{MCAO} / \mathrm{R}$ and $\mathrm{MCAO} / \mathrm{R}+$ EA groups compared to those of the sham group, whereas the numbers of autophagosomes, autolysosomes and lysosomes were increased in both these groups compared to the sham group ( $\mathrm{p}<0.01 ;$ Fig. 3B). However, following treatment with EA for 3 days, in the MCAO/R + EA group, only mild injury to the neurons was visible. Nonetheless, a number of normal organelles and nuclei were still observed. Chromatin was distributed relatively evenly. The autophagosomes, autolysosomes and lysosomes remained present in the cortical neurons in the peri-infact are (Fig. 3A, MCAO/R + EA group). The number of normal mitochondria was increased, whereas the numbers of autophagosomes, autolysosomes and lysosomes were decreased in the rats in the $\mathrm{MCAO} / \mathrm{R}+\mathrm{EA}$ group compared to those in the MCAO/R group ( $<<0.01$; Fig. 3B). 

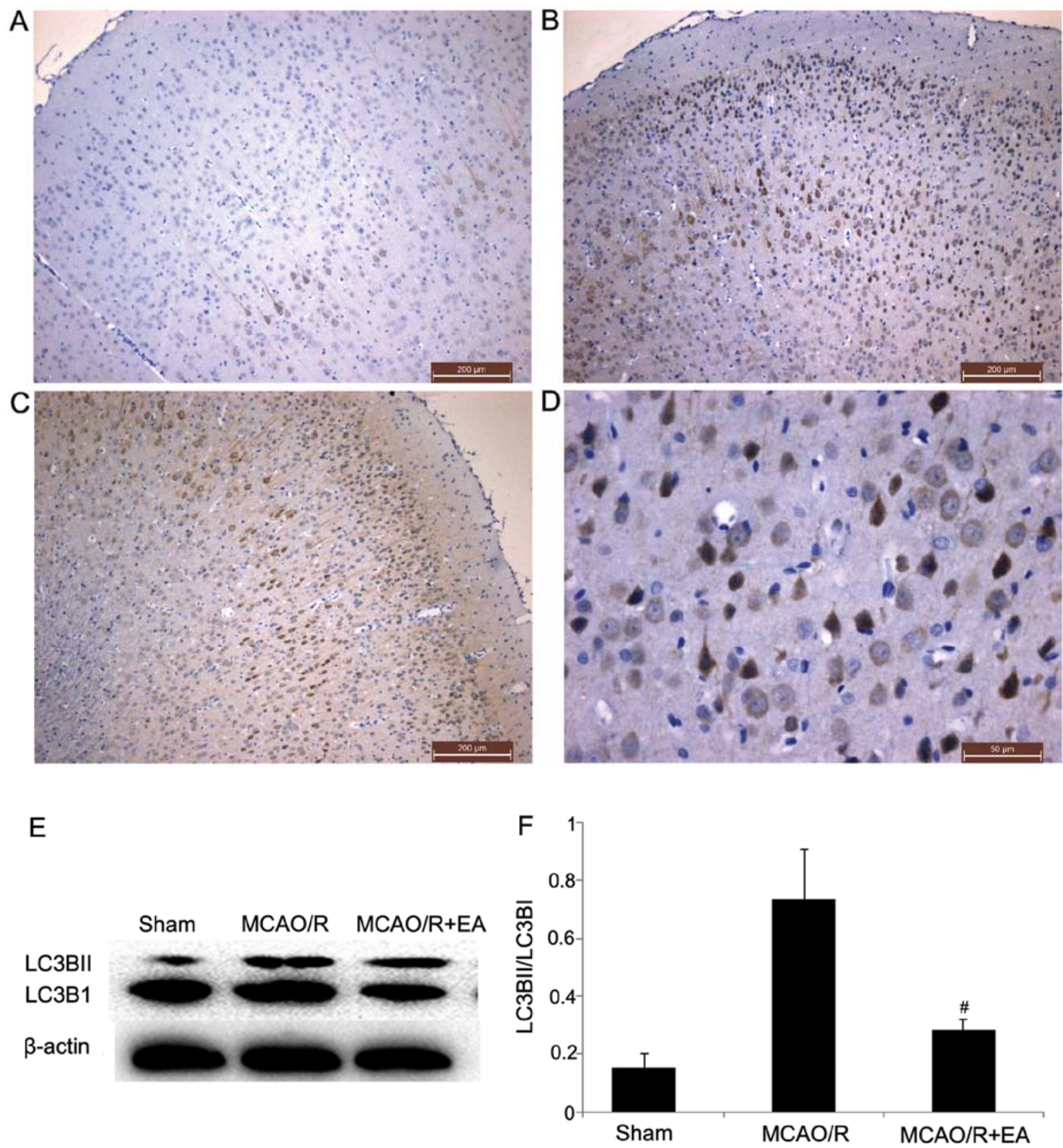

Figure 4. Electroacupuncture (EA) inhibits the expression of autophagosome membrance protein 1B-light chain 3 (LC3B) in the peri-infarct cortex Representative immunohistochemical staining for LC3B protein expression in the peri-infarct cortex. (A) Sham-operated (sham) group; (B) middle cerebral artery occlusion and reperfusion (MCAO/R) group; (C) MCAO/R + EA group. Scale bars, $200 \mu \mathrm{m}$. (D) Amplification of the image in (B). Scale bars, $5 \mu \mathrm{m}$. Western blot analysis of the protein expression levels of LC3B. (E) Representative blots for LC3B expression in each group; (F) Bar graph shows quantitative analysis of the ratios of LC3BII/LC3BI. Data are expressed as the means \pm SEM from 4 individual rats in each group. ** $\mathrm{p}<0.01 \mathrm{MCAO} / \mathrm{R}$ group vs. sham group; " $\mathrm{p}<0.05$, the $\mathrm{MCAO} / \mathrm{R}+\mathrm{EA}$ group vs. the $\mathrm{MCAO} / \mathrm{R}$ group.

Effects of EA on autophagosome membran protein levels in the peri-infarct cortex. Microtubule-associated protein 1A/B LC3 is important for the transport and maturation of autophagosomes. During autophagosome formation, a cytosolic form of LC3 (LC3-I) is conjugated to phosphatidylethanolamine to form LC3-II, which is recruited to autophagosomal membranes. Therefore, LC3-II/LC3-I is considered a biomarker of autophagosome formation and autophagy $(42,43)$. As shown in Fig. 4A-D, in the rats in each group, there was a range of shapes of LC3B brown-yellow-positive cells located in the cytoplasm, such as round-, oval and cone-shaped cells, which were present in the cortical and/or cortical peri-infarct area of granule cells and pyramidal cells. The level of LC3BII/LC3BI was significantly increased in the MCAO/R group compared to the sham group ( $\mathrm{p}<0.01 ;$ Fig. $4 \mathrm{E}$ and F). Following treatment with EA for 3 days, the level of LC3BII/LC3BI was significantly decreased in the $\mathrm{MCAO} / \mathrm{R}+\mathrm{EA}$ group compared to the $\mathrm{MCAO} / \mathrm{R}$ group $(\mathrm{P}<0.05$; Fig. 4E and $\mathrm{F})$. The abovementioned results suggest that EA at the LI11 and ST36 acupoints suppresses autophagosome formation and autophagy in cortical neurons in the peri-infarct area.

Effects of EA on the mTORC1-ULK1 complex-Beclinl pathway. In order to examine the mechanisms involved in the inhibition of 

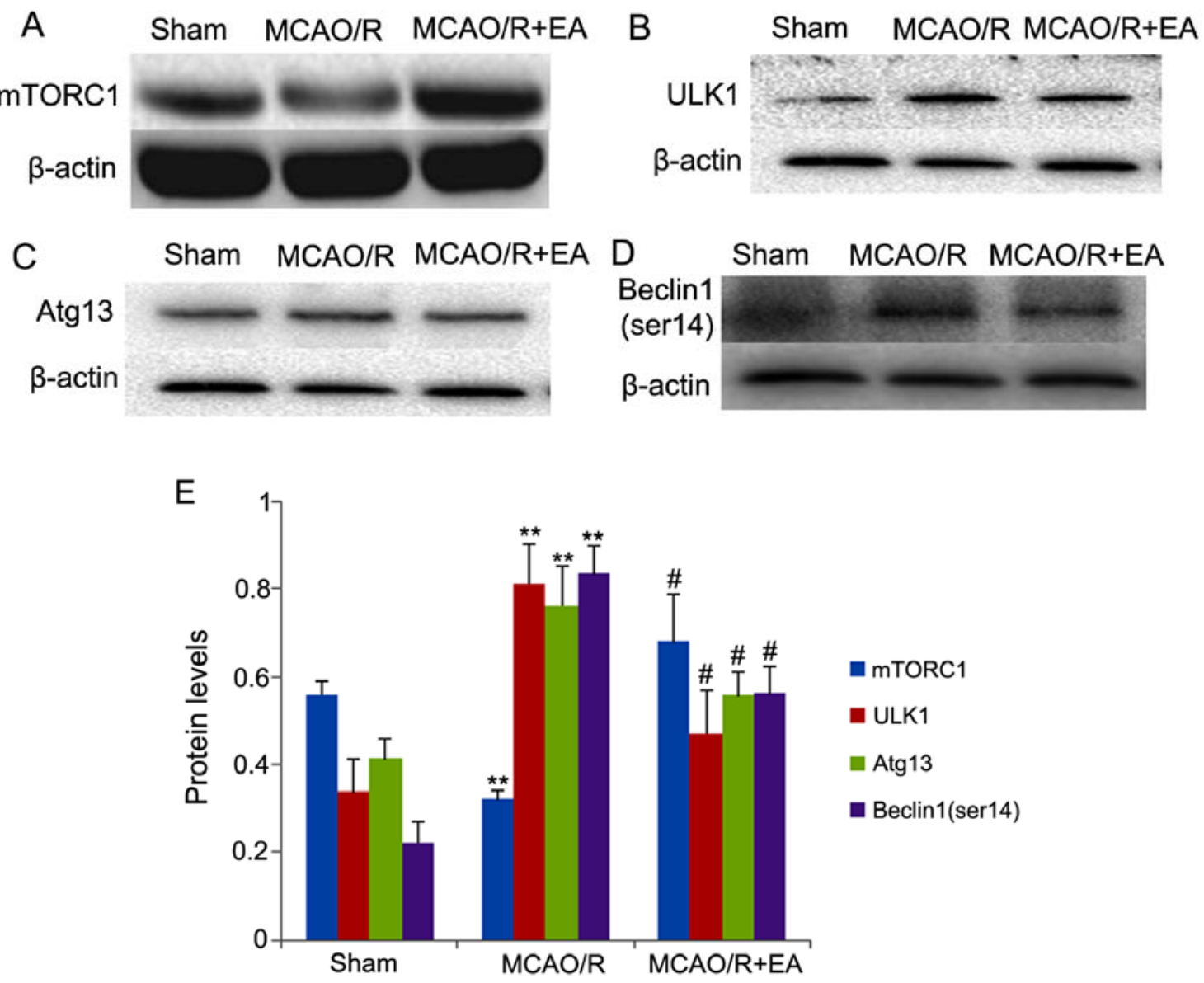

Figure 5. Effects of electroacupuncture (EA) on the mammalian target of rapamycin complex 1 (mTORC1)-Unc-51-like kinase 1 (ULK1)-Beclin1 signaling pathway in the peri-infarct cortex. Western blot analysis of the levels of the mTORC1, ULK1, Atg13 and Beclin1 (ser14) in the peri-infarct cortical tissue of the sham-operated (sham), middle cerebral artery occlusion and reperfusion (MCAO/R) and MCAO/R + EA groups. Representative blots for the (A) mTORC1, (B) ULK1, (C) Atg13 and (D) Beclin1 expression in each group. (E) Bar graph shows quantitative analysis of proteins levels. $\beta$-actin was used as the loading control. Data are expressed as the means \pm SEM from 4 individual rats in each group. ${ }^{* *} \mathrm{p}<0.01$, the MCAO/R group vs. the sham group; ${ }^{*} \mathrm{p}<0.05$, the $\mathrm{MCAO} / \mathrm{R}+\mathrm{EA}$ group vs. the $\mathrm{MCAO} / \mathrm{R}$ group.

autophagosome formation and autophagy by EA, we examined the expression of mTORC1-ULK1 complex-Beclin1 signaling pathway-related proteins. The results are shown in Fig. 5. In the rats in the MCAO/R group, the expression of mTORC1 was significantly decreased compared with that in the rats in the sham group ( $<<0.01$; Fig. 5), whereas the levels of ULK1, Atg13 and Beclin1 (ser14) were significantly increased compared with those in the rats in the sham group ( $\mathrm{p}<0.01 ;$ Fig. 5B-E). However, in the $\mathrm{MCAO} / \mathrm{R}+\mathrm{EA}$ group, treatment with EA significantly increased the expression of mTORCl compared with that in the rats in the $\mathrm{MCAO} / \mathrm{R}$ group ( $\mathrm{p}<0.05$; Fig. $5 \mathrm{~A}$ and $\mathrm{E}$ ), whereas treatment with EA significantly decreased the levels of ULK1, Atg13 and Beclin1 (ser14) compared with those in the MCAO/R group ( $<<0.01$; Fig. 5B-E). Our results indicated that EA at the LI11 and ST36 acupoints markedly increased the expression of mTORC1, leading to the inactivation of the ULK complex and inhibiting the phosphorylation of Beclin1 (ser14) in the periinfarct cortex following MCAO/R injury.

Therefore, the abovementioned results support the hypothesis that EA at the LI11 and ST36 acupoints protects against the harmful effects of ischemic stroke through inhibition of autophagosome formation and autophagy, and these effects are mediated through the mTORC1-ULK complex-Beclin1 pathway.

\section{Discussion}

Thrombosis, embolisms and systemic hypoperfusion can all cause an ischemic stroke. EA, electric stimulation to the acupoints through acupuncture needles, is one of the safest and most effective therapies for ischemic stroke patients, and is a novel therapy based on acupuncture of traditional Chinese medicine (TCM) combined with modern electrotherapy (44-47). EA has served as a complementary therapy and has been noted to improve motor functional outcomes in cases of ischemic stroke $(45,48)$. While reviewing ancient Chinese documents regarding acupuncture and motor impairment, we discovered the LI11 and ST36 were the most frequently selected acupoints in motor rehabilitation treatment in China (49). Animal experimental studies have demonstrated that treatment with EA exerts neuroprotective effects following ischemic stroke $(50,51)$. Consistent with the results of these studies, the results of the present study demonstrated that treatment with EA at the LI11 and ST36 acupoints for 3 consecutive days in rats with MCAO effectively reduced the cerebral infarct volumes and improved neurological deficits. The CatWalk automated gait analysis system has previously been noted to be a sensitive tool for the evaluation of motor function in rat 
models of ischemic stroke $(52,53)$. Significant brain damage has previously been observed in regions controlling the contralateral limbs, leading to a reduction in the paw print area, and intensity and maximum contact area by day 4 post-injury (54). In the present study, using the CatWalk automated gait analysis system, we found that treatment with EA improved the print area and maximum contact area of their four limbs, the stride length of their RF, RH and LF limbs, and the swing speed of their RF, LF and LH limbs at the 4 days after MCAO/R injury, and these results were accompanied by improved neurological deficit outcomes and infarct volumes.

Previous studies have demonstrated that autophagy plays an important role in focal cerebral ischemia $(55,56)$. Published data have shown that cerebral ischemia induces autophagosome formation and autophagy through the activation of the autophagy-related proteins, LC3 and Beclin1 $(57,58)$. Moreover, autophagy has emerged as a potential therapeutic target for the treatment of stroke (59). In a previous study, 3-methyladenine (3-MA) treatment significantly reduced LC3-II conversion and autophagosome formation, which attenuates secondary thalamic damage after focal cerebral infarction (57). Pre-treatment with EA has been shown to promote tolerance against cerebral ischemic injury through the inhibition of LC3-II and Beclin1 expression (60). In the present study, we demonstrated that treatment with EA reduced the number of autophagosomes, autolysosomes and lysosomes, and simultaneously decreased the ratio of LC3BII/LC3BI in the peri-infarct cortex. These results indicate that EA treatment suppresses autophagy following ischemic stroke, which is associated with recovery following brain damage.

In the present study, we further investigated the possible mechanisms involved in the inhibition of autophagy. Increasing evidence suggests that mTOR signaling plays an important role in autophagosome formation and autophagy (19-22). By interacting with other partners, the mTOR kinase forms two functionally distinct complexes, termed mTORC1 and mTORC2. mTORC1 is a central regulator of autophagosome formation and autophagy (61). A number of upstream signaling molecules, including PI3K/Akt, converge on the mTORC1 complex (62). However, inactivated mTORC1 induces autophagy through the coordinated phosphorylation of the ULK complex (ULK1,ULK2 and Atg13) at specific sites (63). Furthermore, previous research has demonstrated that the ULK complex induces autophagy by phosphorylating Beclin1 (26). Importantly, Beclin1 interacts with phosphatidylinositol 3-kinase, class III (PI3KC3), forming Beclin1-PI3KC3 complexes which consist of the mammalian orthologues of class III PI3K, Atg14, Beclin1, hVps15 and $\mathrm{hVps34}$, which are key signaling complexes required for autophagosome formation (64). A marked elevation in Beclin1 levels in the ischemic penumbra of rats following cerebral ischemia has previously been noted 65). The upregulation of Beclin1 together with the increased number of autophagosomes in the ipsilateral hemisphere is likely a response to MCAO reperfusion (66). It has previously been suggested that Beclin1 is important for autophagy in the area surrounding the ischemic core and may be a target for repairing ischemic injury following stroke (67). The results of the present study demonstrated that treatment with EA at the LI11 and ST36 acupoints markedly increased the mTORC1 levels, leading to the inactivation of the ULK complex, and the inhibition of the phosphorylation of Beclin1 (ser14) in the peri-infarct cortex following $\mathrm{MCAO} / \mathrm{R}$ injury, suggesting that EA at the LI11 and ST36 acupoints protected against the damaging effects of ischemic stroke through the inhibition of autophagosome formation and autophagy; these effects were mediated through the mTORC1-ULK complex-Beclin1 pathway.

However, it is still unknown whether increasing, or inhibiting autophagy exerts a neuroprotective effect against cerebral infarction and neurological deficits following ischemic stroke (68). Although autophagy has been examined using animal models of brain ischemia, the majority of the studies have been inconclusive, or have produced contradictory outcomes $(69,70)$. This lack of consensus on the role of autophagy in stroke-induced injury may largely be due to methodological approaches through which autophagy has been either 'activated' or 'inhibited'. In addition, the activation or the inhibition of autophagy is associated with the type of ischemic models and the stage of ischemic stroke. The present study suggested that the inhibition of autophagy in the peri-infarct cortex contributes to the neuroprotective effects induced by EA against focal cerebral ischemia.

In conclusion, our data suggested that EA at the LI11 and ST36 acupoints improved motor dysfunction, and this was accompanied by improved neurological deficit outcomes and decreased infarct volumes following cerebral ischemia and reperfusion. EA at the LI11 and ST36 acupoints protected against the damaning effects of ischemic stroke through the inhibition of autophagosome formation and autophagy, and these effects were mediated through the mTORC1-ULK complex-Beclin1 pathway.

\section{Acknowledgements}

This study was supported by the National Natural Science Foundation of China (nos. 81273835, 81373778 and 81403450).

\section{References}

1. Donnan GA, Fisher M, Macleod M and Davis SM: Stroke. Lancet 371: 1612-1623, 2008.

2. Nour M, Scalzo F and Liebeskind DS: Ischemia-reperfusion injury in stroke. Interv Neurol 1: 185-199, 2013.

3. Zhao H, Sapolsky RM and Steinberg GK: Interrupting reperfusion as a stroke therapy: ischemic postconditioning reduces infarct size after focal ischemia in rats. J Cereb Blood Flow Metab 26: 1114-1121, 2006.

4. Eltzschig HK and Eckle T: Ischemia and reperfusion - from mechanism to translation. Nat Med 17: 1391-1401, 2011.

5. Glick D, Barth S and Macleod KF: Autophagy: cellular and molecular mechanisms. J Pathol 221: 3-12, 2010.

6. Hotchkiss RS, Strasser A, McDunn JE and Swanson PE: Cell death. N Engl J Med 361: 1570-1583, 2009.

7. Martínez-Borra J and López-Larrea C: Autophagy and self-defense. Adv Exp Med Biol 738: 169-184, 2012.

8. Tan CC, Yu JT, Tan MS, Jiang T, Zhu XC and Tan L: Autophagy in aging and neurodegenerative diseases: implications for pathogenesis and therapy. Neurobiol Aging 35: 941-957, 2014.

9. Wen YD, Sheng R, Zhang LS, Han R, Zhang X, Zhang XD, Han F, Fukunaga K and Qin ZH: Neuronal injury in rat model of permanent focal cerebral ischemia is associated with activation of autophagic and lysosomal pathways. Autophagy 4: 762-769, 2008.

10. Shi R, Weng J, Zhao L, Li XM, Gao TM and Kong J: Excessive autophagy contributes to neuron death in cerebral ischemia. CNS Neurosci Ther 18: 250-260, 2012.

11. Wang JY, Xia Q, Chu KT, Pan J, Sun LN, Zeng B, Zhu YJ, Wang Q, Wang $K$ and Luo BY: Severe global cerebral ischemia-induced programmed necrosis of hippocampal CA1 neurons in rat is prevented by 3-methyladenine: a widely used inhibitor of autophagy. J Neuropathol Exp Neurol 70: 314-322, 2011. 
12. Nah J, Yuan J and Jung YK: Autophagy in neurodegenerative diseases: from mechanism to therapeutic approach. Mol Cells 38: 381-389, 2015.

13. Mochida K, Oikawa Y, Kimura Y, Kirisako H, Hirano H, Ohsumi Y and Nakatogawa H: Receptor-mediated selective autophagy degrades the endoplasmic reticulum and the nucleus. Nature 522: 359-362, 2015.

14. Tanida I: Autophagosome formation and molecular mechanism of autophagy. Antioxid Redox Signal 14: 2201-2214, 2011.

15. Tanida I, Ueno T and Kominami E: LC3 and Autophagy. Methods Mol Biol 445: 77-88, 2008

16. Mizushima N, Yoshimori T and Ohsumi Y: The role of Atg proteins in autophagosome formation. Annu Rev Cell Dev Biol 27: 107-132, 2011.

17. Dooley HC, Razi M, Polson HE, Girardin SE, Wilson MI and Tooze SA: WIPI2 links LC3 conjugation with PI3P, autophagosome formation, and pathogen clearance by recruiting Atg12-5-16L1. Mol Cell 55: 238-252, 2014.

18. Kim YM, Jung CH, Seo M, Kim EK, Park JM, Bae SS and Kim DH: mTORC1 phosphorylates UVRAG to negatively regulate autophagosome and endosome maturation. Mol Cell 57: 207-218, 2015.

19. Hosokawa N, Hara T, Kaizuka T, Kishi C, Takamura A, Miura Y, Iemura S, Natsume T, Takehana K, Yamada N, et al: Nutrient-dependent mTORC1 association with the ULK1Atg13-FIP200 complex required for autophagy. Mol Biol Cell 20: 1981-1991, 2009.

20. Szymańska P, Martin KR, MacKeigan JP, Hlavacek WS and Lipniacki T: Computational analysis of an autophagy/translation switch based on mutual inhibition of MTORC1 and ULK1. PLoS One 10: e0116550, 2015.

21. Denton D, Nicolson S and Kumar S: Cell death by autophagy: facts and apparent artefacts. Cell Death Differ 19: 87-95, 2012.

22. Puyal J, Vaslin A, Mottier V and Clarke PG: Postischemic treatment of neonatal cerebral ischemia should target autophagy. Ann Neurol 66: 378-389, 2009.

23. Wei K, Wang P and Miao CY: A double-edged sword with therapeutic potential: an updated role of autophagy in ischemic cerebral injury. CNS Neurosci Ther 18: 879-886, 2012.

24. Rami A and Kögel D: Apoptosis meets autophagy-like cell death in the ischemic penumbra: two sides of the same coin? Autophagy 4: 422-426, 2008.

25. Tanida I: Autophagy basics. Microbiol Immunol 55: 1-11, 2011.

26. Russell RC, Tian Y, Yuan H, Park HW, Chang YY, Kim J, Kim H, Neufeld TP, Dillin A and Guan KL: ULK1 induces autophagy by phosphorylating Beclin-1 and activating VPS34 lipid kinase. Nat Cell Biol 15: 741-750, 2013.

27. Nazarko VY and Zhong Q: ULK1 targets Beclin-1 in autophagy. Nat Cell Biol 15: 727-728, 2013.

28. Chen A, Lin Z, Lan L, Xie G, Huang J, Lin J, Peng J, Tao J and Chen L: Electroacupuncture at the Quchi and Zusanli acupoints exerts neuroprotective role in cerebral ischemia-reperfusion injured rats via activation of the PI3K/Akt pathway. Int J Mol Med 30: 791-796, 2012.

29. Xue X, You Y, Tao J, Ye X, Huang J, Yang S, Lin Z, Hong Z Peng $J$ and Chen L: Electro-acupuncture at points of Zusanli and Quchi exerts anti-apoptotic effect through the modulation of PI3K/Akt signaling pathway. Neurosci Lett 558: 14-19, 2014.

30. Du F and Liu S: Electroacupuncture with high frequency at acupoint ST-36 induces regeneration of lost enteric neurons in diabetic rats via GDNF and PI3k/Akt signal pathway. Am J Physiol Regul Integr Comp Physiol 309: R109-R118, 2015.

31. Wu YT, Tan HL, Huang Q, Ong CN and Shen HM: Activation of the PI3K-Akt-mTOR signaling pathway promotes necrotic cell death via suppression of autophagy. Autophagy 5: 824-834, 2009.

32. Heras-Sandoval D, Pérez-Rojas JM, Hernández-Damián J and Pedraza-Chaverri J: The role of PI3K/AKT/mTOR pathway in the modulation of autophagy and the clearance of protein aggregates in neurodegeneration. Cell Signal 26: 2694-2701, 2014.

33. Longa EZ, Weinstein PR, Carlson S and Cummins R: Reversible middle cerebral artery occlusion without craniectomy in rats. Stroke 20: 84-91, 1989.

34. Huang J, Ye X, You Y, Liu W, Gao Y, Yang S, Peng J, Hong Z, Tao J and Chen L: Electroacupuncture promotes neural cell proliferation in vivo through activation of the ERK1/2 signaling pathway. Int J Mol Med 33: 1547-1553, 2014.

35. Neumann M, Wang Y, Kim S, Hong SM, Jeng L, Bilgen M and Liu J: Assessing gait impairment following experimental traumatic brain injury in mice. J Neurosci Methods 176: 34-44, 2009.
36. Encarnacion A, Horie N, Keren-Gill H, Bliss TM, Steinberg GK and Shamloo M: Long-term behavioral assessment of function in an experimental model for ischemic stroke. J Neurosci Methods 196: 247-257, 2011.

37. Wang Y, Yoshimura R, Manabe H, Schretter C, Clarke R, Cai Y, Fitzgerald $\mathrm{M}$ and Lee KS: Trans-sodium crocetinate improves outcomes in rodent models of occlusive and hemorrhagic stroke. Brain Res 1583: 245-254, 2014.

38. Liu Y, Ao LJ, Lu G, Leong E, Liu Q, Wang XH, Zhu XL, Sun TF, Fei Z, Jiu T, et al: Quantitative gait analysis of long-term locomotion deficits in classical unilateral striatal intracerebral hemorrhage rat model. Behav Brain Res 257: 166-177, 2013.

39. Koopmans GC, Deumens R, Honig WM, Hamers FP, Steinbusch HW and Joosten EA: The assessment of locomotor function in spinal cord injured rats: the importance of objective analysis of coordination. J Neurotrauma 22: 214-225, 2005.

40. Dupont N, Orhon I, Bauvy C and Codogno P: Autophagy and autophagic flux in tumor cells. Methods Enzymol 543: 73-88, 2014.

41. Mauvezin C, Ayala C, Braden CR, Kim J and Neufeld TP: Assays to monitor autophagy in Drosophila. Methods 68: 134-139, 2014.

42. Kabeya Y, Mizushima N, Ueno T, Yamamoto A, Kirisako T, Noda T, Kominami E, Ohsumi Y and Yoshimori T: LC3, a mammalian homologue of yeast Apg8p, is localized in autophagosome membranes after processing. EMBO J 19: 5720-5728, 2000.

43. Li YC, He SM, He ZX, Li M, Yang Y, Pang JX, Zhang X, Chow K, Zhou Q, Duan W, et al: Plumbagin induces apoptotic and autophagic cell death through inhibition of the PI3K/Akt/mTOR pathway in human non-small cell lung cancer cells. Cancer Lett 344: 239-259, 2014.

44. Li X, Luo P, Wang Q and Xiong L: Electroacupuncture pretreatment as a novel avenue to protect brain against ischemia and reperfusion injury. Evid Based Complement Alternat Med 2012: 195397, 2012.

45. Liu SY, Hsieh CL, Wei TS, Liu PT, Chang YJ and Li TC: Acupuncture stimulation improves balance function in stroke patients: a single-blinded controlled, randomized study. Am J Chin Med 37: 483-494, 2009.

46. Wayne PM, Krebs DE, Macklin EA, Schnyer R, Kaptchuk TJ, Parker SW, Scarborough DM, McGibbon CA, Schaechter JD, Stein J and Stason WB: Acupuncture for upper-extremity rehabilitation in chronic stroke: a randomized sham-controlled study. Arch Phys Med Rehabil 86: 2248-2255, 2005.

47. Zhang GC, Fu WB, Xu NG, Liu JH, Zhu XP, Liang ZH, Huang YF and Chen YF: Meta analysis of the curative effect of acupuncture on post-stroke depression. J Tradit Chin Med 32: 6-11, 2012.

48. Lim SM, Yoo J, Lee E, Kim HJ, Shin S, Han G and Ahn HS: Acupuncture for spasticity after stroke: a systematic review and meta-analysis of randomized controlled trials. Evid Based Complement Alternat Med 2015: 870398, 2015.

49. Yang HY, Liu TY, Wang YH, Ying SG, Zheng CY, Kuai L and Gao M: Acupoint electrogymnastics therapy for treatment of apoplectic hemiplegia: a multicenter randomized control study. Zhongguo Zhen Jiu 28: 635-638, 2008 (In Chinese).

50. Feng X, Yang S, Liu J, Huang J, Peng J, Lin J, Tao J and Chen L: Electroacupuncture ameliorates cognitive impairment through inhibition of NF- $\mathrm{kB}$-mediated neuronal cell apoptosis in cerebral ischemia-reperfusion injured rats. Mol Med Rep 7: 1516-1522, 2013.

51. Zhang TS, Yang L, Hu R, Qiao XL, Yang X and Liu XG: Effect of electroacupuncture on the contents of excitatory amino acids in cerebral tissue at different time courses in rats with cerebral ischemia and reperfusion injury. Zhen Ci Yan Jiu 32: 234-236, 2007 (In Chinese).

52. Vandeputte C, Taymans JM, Casteels C, Coun F, Ni Y, Van Laere $\mathrm{K}$ and Baekelandt V: Automated quantitative gait analysis in animal models of movement disorders. BMC Neurosci 11: 92, 2010.

53. Lubjuhn J, Gastens A, von Wilpert G, Bargiotas P, Herrmann O, Murikinati S, Rabie T, Marti HH, Amende I, Hampton TG and Schwaninger M: Functional testing in a mouse stroke model induced by occlusion of the distal middle cerebral artery. $\mathrm{J}$ Neurosci Methods 184: 95-103, 2009.

54. Wang Y, Bontempi B, Hong SM, Mehta K, Weinstein PR, Abrams GM and Liu J: A comprehensive analysis of gait impairment after experimental stroke and the therapeutic effect of environmental enrichment in rats. J Cereb Blood Flow Metab 28: 1936-1950, 2008. 
55. Xu M and Zhang HL: Death and survival of neuronal and astrocytic cells in ischemic brain injury: a role of autophagy. Acta Pharmacol Sin 32: 1089-1099, 2011.

56. Xu F, Gu JH and Qin ZH: Neuronal autophagy in cerebral ischemia. Neurosci Bull 28: 658-666, 2012.

57. Xing S, Zhang Y, Li J, Zhang J, Li Y, Dang C, Li C, Fan Y, Yu J, Pei Z and Zeng J: Beclin 1 knockdown inhibits autophagic activation and prevents the secondary neurodegenerative damage in the ipsilateral thalamus following focal cerebral infarction. Autophagy 8: 63-76, 2012.

58. Rami A, Langhagen A and Steiger S: Focal cerebral ischemia induces upregulation of Beclin 1 and autophagy-like cell death. Neurobiol Dis 29: 132-141, 2008.

59. Levine B, Packer M and Codogno P: Development of autophagy inducers in clinical medicine. J Clin Invest 125: 14-24, 2015.

60. Wu Z, Zou Z, Zou R, Zhou X and Cui S: Electroacupuncture pretreatment induces tolerance against cerebral ischemia/reperfusion injury through inhibition of the autophagy pathway. Mol Med Rep 11: 4438-4446, 2015.

61. Inoki K, Kim J and Guan KL: AMPK and mTOR in cellular energy homeostasis and drug targets. Annu Rev Pharmacol Toxicol 52: 381-400, 2012.

62. Leung EY, Askarian-Amiri M, Finlay GJ, Rewcastle GW and Baguley BC: Potentiation of growth inhibitory responses of the mTOR inhibitor everolimus by dual mTORC $1 / 2$ inhibitors in cultured breast cancer cell lines. PLoS One 10: e0131400, 2015.

63. Zha QB, Zhang XY, Lin QR, Xu LH, Zhao GX, Pan H, Zhou D, Ouyang DY, Liu ZH and He XH: Cucurbitacin E induces autophagy via downregulating mTORC1 signaling and upregulating AMPK activity. PLoS One 10: e0124355, 2015.
64. Wirth M, Joachim J and Tooze SA: Autophagosome formation - the role of ULK1 and Beclin1-PI3KC3 complexes in setting the stage. Semin Cancer Biol 23: 301-309, 2013.

65. Rami A: Upregulation of Beclin 1 in the ischemic penumbra. Autophagy 4: 227-229, 2008.

66. Zheng YQ, Liu JX, Li XZ, Xu L and Xu YG: RNA interferencemediated downregulation of Beclin1 attenuates cerebral ischemic injury in rats. Acta Pharmacol Sin 30: 919-927, 2009.

67. Klionsky DJ, Abdalla FC, Abeliovich H, Abraham RT, Acevedo-Arozena A, Adeli K, Agholme L, Agnello M, Agostinis P, Aguirre-Ghiso JA, et al: Guidelines for the use and interpretation of assays for monitoring autophagy. Autophagy 8: 445-544, 2012

68. Gao L, Jiang T, Guo J, Liu Y, Cui G, Gu L, Su L and Zhang Y: Inhibition of autophagy contributes to ischemic postconditioninginduced neuroprotection against focal cerebral ischemia in rats. PLoS One 7: e46092, 2012.

69. Buckley KM, Hess DL, Sazonova IY, Periyasamy-Thandavan S, Barrett JR, Kirks R, Grace H, Kondrikova G, Johnson MH, Hess DC, et al: Rapamycin up-regulation of autophagy reduces infarct size and improves outcomes in both permanent MCAL, and embolic MCAO, murine models of stroke. Exp Transl Stroke Med 6: 8, 2014

70. Zheng Y, Hou J, Liu J, Yao M, Li L, Zhang B, Zhu H and Wang Z: Inhibition of autophagy contributes to melatonin-mediated neuroprotection against transient focal cerebral ischemia in rats. J Pharmacol Sci 124: 354-364, 2014. 